\title{
Determinação da equação intensidade-duração-frequência para três estações meteorológicas do Estado de Mato Grosso ${ }^{1}$
}

\author{
Samantha S. G arcia ${ }^{2}$, Ricardo S. S. Amorim ${ }^{3}$, Eduardo G. Couto ${ }^{3} \&$ W erico H. Stopa ${ }^{4}$
}

\begin{abstract}
RESUMO
O bjetivou-se, com este trabalho, determinar a equação intensidade-duração-frequência para três estações meteorológicas pertencentes à rede hidrometeorológica do Instituto $\mathrm{N}$ acional de M eteorologia (INMET), no Estado de Mato Grosso (Cáceres, Cuiabá e Rondonópolis) utilizando-se as metodologias de análise de pluviogramas, desagregação de chuvas de $24 \mathrm{~h}$ e de Bell e comparar as estimativas da intensidade de precipitação aplicando-se as equações obtidas através dessas metodologias. Dados pluviográficos e pluviométricos foram utilizados para obtenção da equação de chuvas intensas. As séries históricas de intensidades máximas médias de precipitação, correspondentes às diversas durações, foram submetidas à análise estatística a fim de identificar o modelo probabilístico que apresentasse melhor ajuste aos dados, sendo esta identificação realizada utilizando-se o teste de aderência de Kolmogorov-Smirnov a nível de significância de $5 \%$. 0 método da Desagregação não só apresentou melhor desempenho global $(E R M=27 \%)$ em comparação ao de Bell $(E R M=31 \%)$, como possui melhor desempenho em estações com séries mais longas mostrando-se, assim, mais sensível ao tamanho da série em relação ao método de Bell.
\end{abstract}

Palavras-chave: chuvas intensas, desagregação de chuvas, método de Bell

\section{Determination of the intensity-duration-frequency equation for three meteorological stations in Mato Grosso State}

\begin{abstract}
The main objective of this study was to determine the intensity-duration-frequency equation for three meteorological stations (Cáceres, Cuiabá and Rondonópolis) belonging to the hydrometeorological network of the National Institute of Meteorology (INMET) in Mato Grosso using the methodologies of pluviograms analysis, daily rain disaggregation and Bell, as well as comparing the estimates of intensity of precipitation by applying the equations obtained through these methodologies. Pluviometric and pluviographic data were used to obtain intense precipitation equation. The historical series of average maximum intensity of precipitation, corresponding to the different durations were submitted to statistical analysis to identify the probabilistic model that presented best fit for the data, this identification being performed using the adherence test of Kolmogorov-Smirnov at $5 \%$ level of significance. The methodology of the Disaggregation not only presented better overall performance (ERM $=27 \%$ ) in comparison to the one of Bell (ERM $=31 \%$ ), as it has better performance in stations with longer series, revealing, thus, more sensitive to the size of the series in relation to the Bell's method.
\end{abstract}

Key words: intense precipitation, daily rain disaggregation, Bell's method

\footnotetext{
1 Parte de Dissertação de Mestrado da primeira autora apresentada ao PPGRH/ICET/UFMT, Cuiabá, MT.

2 Engenheira Agrônoma. Av. Fernando Correa da Costa n. 2367, Campus U FMT. Cuiabá, MT. CEP 78060-900. Fone/Fax: (65) 3615 8616. E-mail: samsgarcia@yahoo.com.br

3 DSER/U FM T. CEP 78060-900. Fone/Fax: (65) 3615-8616/8668. E-mail: rsamorim@ufmt.br; couto@ufmt.br

${ }^{4}$ Graduando em Agronomia da FAMEV/U FMT. E-mail: werico-stopa@hotmail.com
} 


\section{INTRODUÇÃO}

O conhecimento de dados de vazão máxima observados é importante por serem utilizados em projetos de obras hidráulicas, tais como vertedouros de barragens, canais de terraços, drenagem agrícola, urbana e rodoviária, desvio de cursos d'água e práticas mecânicas de controle de erosão hídrica, entre outros.

Um dos caminhos que permitem conhecer a vazão de projeto quando não se tem dados históricos de vazão ou mesmo quando se apresentam em menor quantidade, é fazer um estudo de chuvas intensas da região.

Esta exigência é atendida, geralmente, a partir de informações pontuais, ou seja, são utilizadas equações do tipo intensidadeduração-frequência (equações de chuvas intensas) derivadas de pluviógrafos específicos da região em estudo (Cecílio \& Pruski, 2003).

Algumas dificuldades constantemente enfrentadas pelos técnicos são: a inexistência daquela equação na localidade onde será realizado o projeto, além de um exaustivo trabalho de análise, interpretação e codificação de grande quantidade de dados.

Desta forma, além dos ajustes da equação intensidadeduração-frequência para as estações meteorológicas providas de dados pluviográficos, torna-se de fundamental importância ajustar uma metodologia que possibilite a determinação desta equação a partir de dados pluviométricos, em função da maior disponibilidade de estação e maior tempo de observações (Mello et al., 2001).

Conforme Oliveira et al. (2008), no Brasil foram desenvolvidas algumas metodologias com vista à obtenção de chuvas de menor duração a partir de registros pluviométricos, devido à existência, no território nacional, de uma vasta rede pluviométrica, dentre as quais se pode citar: o método de desagregação de chuvas de 24 h e o método de Bell.

Oliveira et al. (2005) obtiveram relações intensidade-duraçãofrequência para algumas localidades do Estado de Goiás e Distrito Federal, empregando a metodologia da desagregação da chuva de um dia e comparando-as com as obtidas pelas equações ajustadas com base em dados de pluviogramas. Nesta comparação as relações geradas com o método de desagregação de chuvas de um dia apresentaram desvios relativos médios que variaram de $-1,6$ a $43,9 \%$, para alguns municípios da região estudada.

No sul do Brasil muitos trabalhos têm sido desenvolvidos por Damé et al. (2006; 2008) a fim de se obter informações sobre o método da desagregação de chuva de $24 \mathrm{~h}$ para as condições locais, estabelecendo-se comparações entre o referido método e o de análise de pluviogramas, através do cálculo de erro padrão da estimativa (EPE).

Svensson et al. (2007), ressaltaram que o cálculo das relações de intensidade, duração e frequência (IDF) para as chuvas, exige registros de precipitação acumulada em períodos mais curtos do que um dia, quando a aplicação é para pequenas bacias e/ou urbanas.

No trabalho referente a Damé et al. (2006), foram encontrados valores discrepantes de intensidade máxima obtidos com a equação IDF e o Método das Relações; já no segundo trabalho, Damé et al. (2008) concluíram que o método que representou melhor as intensidades máximas foi o Método das Relações, quando comparado com outros três métodos.

Mello et al. (2003), ajustaram o método de Bell para diferentes regiões do Estado de Minas Gerais tomando como base a chuva máxima, com duração de 1 h e período de retorno de 2 anos, e concluíram que o Estado está dividido entre regiões, com base em características fisiográficas, pluviométricas e tipos climáticos, apresentando boa homogeneidade, em termos de precipitação intensa.

Para Oliveira et al. (2008), o método de Bell se ajustou perfeitamente aos dados dos municípios estudados no Estado de Goiás, apresentando-se como alternativa na obtenção das alturas de chuvas intensas a partir de séries curtas.

Back (2008), analisou pluviogramas da estação meteorológica de Urussanga, SC, para, com o objetivo de ajustar os coeficientes de Bell cuja base foi a chuva máxima, com período de retorno de 10 anos e duração de 1 dia. $O$ autor afirma que as relações obtidas com base na chuva máxima com duração de um dia e período de retorno de 10 anos, apresentam erro padrão da estimativa equivalente às obtidas com base na análise de pluviogramas evidenciando, desta forma, que método de Bell pode ser uma alternativa na determinação de equações de chuva intensas para locais sem registro de dados pluviográficos; apesar disto, Back apresentou, no mesmo ano, algumas limitações para a metodologia de Bell, tais como: suas equações foram obtidas a partir de dados de chuva de diversas partes do mundo; portanto, os resultados são obtidos em função de valores médios e não específicos para um local; o valor da precipitação máxima obtida é válido apenas para durações entre 5 e 120 min, enquanto a necessidade de se conhecer a chuva máxima com duração de 1 h e período de retorno de 10 anos, é fato que requer dados de estação com pluviógrafo.

Desta forma, o presente estudo tem como objetivo geral de determinar as equações intensidade-duração-frequência para três estações meteorológicas da região centro sul do Estado de Mato Grosso, contemplando os seguintes objetivos específicos: estabelecer a relação entre intensidade, duração e frequência da precipitação pluvial, a partir dos registros pluviográficos das referidas estações, através das metodologias de análise de pluviogramas, de desagregação de chuvas de 24 h e de Bell; ajustar modelos teóricos de distribuição de probabilidade aos dados de chuvas intensas de 3 estações pluviográficas localizadas no Estado de Mato Grosso; estimar equações de chuvas intensas para essas mesmas estações meteorológicas empregando as três metodologia já referidas; comparar as intensidades de precipitação estimadas pelas equações obtidas pela análise de pluviogramas, pela desagregação da chuva de 24 h e pelo método de Bell.

\section{Material e MÉTOdos}

Para a realização do presente trabalho foram utilizados dados de três estações pluviográficas localizadas em três municípios (Cáceres, Cuiabá e Rondonópolis) do Estado de Mato Grosso pertencentes à rede hidrometeorológica do Instituto Nacional de Meteorologia (INMET) (Tabela 1 e Figura 1). 
Tabela 1. Localidades do Estado de M ato Grosso utilizadas no presente estudo

\begin{tabular}{lccccc}
\hline Localidades & Código & Latitude & Longitude & Altitude & Período de observação (anos) \\
Cáceres & 01657003 & $-16,1308$ & $-57,7936$ & $118,00 \mathrm{~m}$ & $1990-1995 ; 1997-1999 ; 2001 ; 2003 ; 2005$ (12 anos) \\
Cuiabá & 01556009 & $-15,6216$ & $-56,1083$ & $151,34 \mathrm{~m}$ & $1972-1977 ; 1985 ; 1990-1999 ; 2003(18$ anos) \\
Rondonópolis & 01654002 & $-16,4500$ & $-54,5666$ & $284,00 \mathrm{~m}$ & $1992-1993,1995 ; 1997-1999 ; 2005$ (7 anos) \\
\hline
\end{tabular}

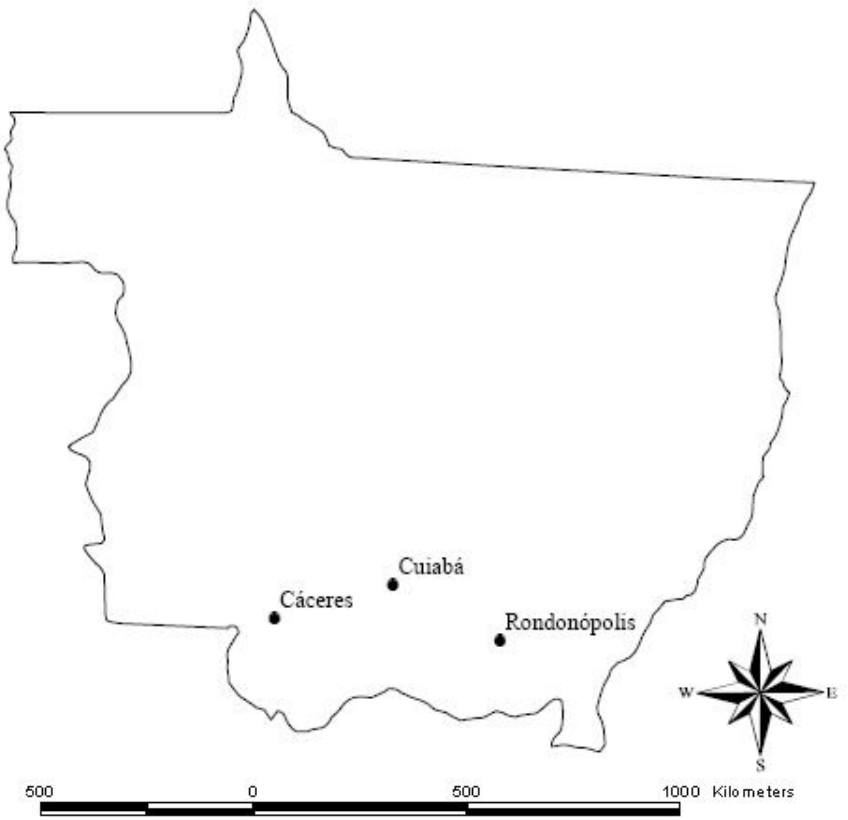

Figura 1. Distribuição espacial das estações meteorológicas do Estado de M ato Grosso utilizadas no presente estudo

A fase de aquisição dos dados de precipitação se constituiu, inicialmente, na seleção dos pluviogramas que continham as chuvas mais intensas observadas em cada ano da série de dados para cada uma das estações pluviográficas a serem estudadas.

Não se adotou um período-base de estudos para todas as estações, visto que elas não tinham períodos coincidentes de observação, sendo as séries históricas anuais de cada estação utilizadas sem o preenchimento de falhas.

Após a seleção dos pluviogramas realizou-se sua leitura com o objetivo de obter as alturas precipitadas máximas anuais para a duração de 10, 20, 30, 40, 50, 60, 120, 180, 240, 360, 720 e $1440 \mathrm{~min}$, em cada uma das estações selecionadas. Dividindo as alturas máximas precipitadas pela duração correspondente, obteve-se a intensidade máxima média de precipitação. Esses valores máximos das precipitações diárias permitiram, assim, a construção das séries anuais dos valores extremos para as três estações selecionadas.

A leitura dos pluviogramas foi realizada no formato digital com uso do Sistema para Digitalização de Pluviogramas (HidroGraph 1.02), desenvolvido pelo Grupo de Pesquisa em Recursos Hídricos do Departamento de Engenharia Agrícola da Universidade Federal de Viçosa, para a Agência Nacional de Águas (ANA).

Com a finalidade de se obter as precipitações máximas possíveis de serem igualadas ou superadas a cada 2, 5, 10, 20, 50 e 100 anos, a partir das séries anuais, empregou-se a distribuição de Gumbel (Eq. 1) e se avaliou sua aderência a cada série de dados pelo teste de Kolmogorov-Smirnov a nível de significância de $5 \%$ de probabilidade.

$$
\mathrm{P}_{\mathrm{x}}=1-\exp \left[-\exp \left(-\mathrm{Y}_{\mathrm{TR}}\right)\right]
$$

em que:

$\mathrm{P}_{\mathrm{x}}$ - probabilidade de um valor extremo da série ser igual ou maior à magnitude de determinado evento

$\mathrm{Y}_{\mathrm{TR}}$ - variável reduzida.

A variável reduzida da distribuição de Gumbel é obtida pela aplicação da função de distribuição de frequência de Chow, dada pelas Eqs. 2 a 4.

$$
\begin{gathered}
\mathrm{Y}_{\mathrm{TR}}=-\ln \left[-\ln \left(1-\frac{1}{\mathrm{Y}_{\mathrm{TR}}}\right)\right] \\
\mathrm{K}_{\mathrm{TR}}=-0,45+0,78 \times \mathrm{Y}_{\mathrm{TR}} \\
\mathrm{X}_{\mathrm{TR}}=\overline{\mathrm{X}}+\mathrm{K}_{\mathrm{TR}} \times \mathrm{S}
\end{gathered}
$$

sendo:

$\mathrm{Y}_{\mathrm{TR}}$ - variável reduzida da distribuição de Gumbel

TR - período de retorno, ano

$\mathrm{K}_{\mathrm{TR}}$ - fator de frequência, adimensional

$\mathrm{X}_{\mathrm{TR}}$ - precipitação máxima diária para determinado $\mathrm{TR}, \mathrm{mm}$

$\bar{X}$ - média dos valores extremos da série histórica de precipitação

S - desvio-padrão dos valores extremos da série histórica de precipitação

Através do método de regressão não linear Gauss-Newton e com base nos valores de intensidade de precipitação máxima, correspondentes aos períodos de retorno de 2, 5, 10, 20, 50 e 100 anos e duração de 5, 10, 15, 20, 25, 30, 60, 360, 420, 600, 720, 1440 min, obtiveram-se os parâmetros da equação de intensidade-duração-frequência (Eq. 5). Este procedimento foi realizado para cada estação pluviográfica com o auxílio do programa computacional Statistica (versão 6.1).

$$
i_{m}=\frac{K T R{ }^{a}}{(t+b)^{c}}
$$

em que:

$$
\begin{aligned}
& \mathrm{i}_{\mathrm{m}} \text { - intensidade máxima média da chuva, } \mathrm{mm} \mathrm{h}^{-1} \\
& \mathrm{TR} \text { - período de retorno, anos } \\
& \mathrm{t} \text { - duração da chuva, min } \\
& \mathrm{k}, \mathrm{a}, \mathrm{b}, \mathrm{c} \text { - coeficientes de ajustamento específicos para cada }
\end{aligned}
$$
localidade. 
Obtenção das precipitações máximas através do método da desagregação de chuvas de $24 \mathrm{~h}$

Pela cotagem dos pluviogramas da série histórica de cada estação obteve-se uma série anual de valores extremos de precipitação diária.

A partir desses dados foram obtidos valores de precipitação com duração de 5, 10, 15, 20, 25, 30, 60, 360, 420, 600, 720, 1440 min, por meio da técnica de desagregação da chuva, proposta por DAEE-CETESB (1980), o que permitiu gerar pontos suficientes para definir as curvas de intensidade-duraçãofrequência, referentes a diferentes períodos de retorno (TR).

$\mathrm{O}$ ajuste das distribuições de probabilidade e a realização do teste de Kolmogorov-Smirnov (5\% de significância) foi realizado utilizando-se o programa Statistica (versão 7.0).

\section{Obtenção das precipitações máximas utilizando-se o método de Bell}

O método de Bell associa a altura pluviométrica de uma chuva intensa para um tempo de duração e período de retorno padrão de 60 min e dois anos, conforme descrito na Eq. 6.

$$
\mathrm{h}_{(\mathrm{t} ; \mathrm{TR})}=\left(\alpha \times \ln (\mathrm{TR})+\beta_{1}\right)+\left(\beta_{2} \times \mathrm{t}^{\gamma}-\beta_{3}\right) \times \mathrm{h}_{(60 ; 2)}
$$

em que:

$\mathrm{h}_{(\mathrm{t} ; \mathrm{TR})}$ - altura pluviométrica $(\mathrm{mm})$ de chuva intensa de duração "t" e período de retorno "TR"

$\mathrm{h}_{(60 ; 2)}$ - altura pluviométrica (mm) de chuva intensa, padrão de 60 min de duração e dois anos de período de retorno,

$\alpha, \gamma, \beta_{1}, \beta_{2}$ e $\beta_{3}$ - parâmetros regionais ajustados pelo método dos mínimos quadrados

Bertoni \& Tucci (1993), recomendam que o método de Bell só se aplica à chuvas com durações menores que $120 \mathrm{~min}$ e períodos de retorno inferiores a 20 anos.

Seguindo esta recomendação determinaram-se as intensidades e alturas pluviométricas máximas para os tempos de duração de $5 ; 10 ; 15 ; 20 ; 25 ; 30 ; 40 ; 50 ; 60$ e 120 min e períodos de retorno de $2 ; 5 ; 10$ e 20 anos.

A equação utilizada (Eq.7) foi ajustada por Bell com dados de vários continentes:

$\mathrm{h}_{(\mathrm{t} ; \mathrm{TR})}=(0,35 \times \ln (\mathrm{TR})+0,76)+\left(0,54 \times \mathrm{t}^{0,25}-0,50\right) \times \mathrm{h}_{(60 ; 2)}(7)$

em que:

$\mathrm{P}$ é a precipitação estimada $(\mathrm{mm})$ de chuva intensa de duração " $t$ " e período de retorno "TR".

\section{Comparação dos métodos de obtenção das equações intensidade-duração-frequência}

Os resultados obtidos pelas equações de chuvas intensas ajustadas pelos métodos alternativos (desagregação de chuvas de 24 h e Bell) foram comparados com os obtidos pelo método padrão (análise de pluviogramas) por meio do erro médio relativo (EMR):

$$
\mathrm{EMR}=100 . \sum \frac{\left|\mathrm{i}_{\mathrm{m}}^{\mathrm{ap}}-\mathrm{i}_{\mathrm{m}}^{\mathrm{dc}}\right|}{\mathrm{i}_{\mathrm{m}}^{\mathrm{ap}}}
$$

em que:

$\mathrm{i}_{\mathrm{m}}^{\mathrm{ap}}$ - intensidades máximas de precipitação obtidas pelas equações ajustadas em função da análise de pluviogramas

$\mathrm{i}_{\mathrm{m}}^{\mathrm{dc}}$ - intensidades máximas de precipitação obtidas pelas equações ajustadas empregado-se a técnica de desagregação de chuvas diárias.

\section{RESULTADOS E DISCUSSÃO}

Os valores de intensidades máximas diárias de precipitação, apresentados na Tabela 2, foram obtidos pelo método de análise de pluviogramas. Dentre as estações pluviográficas estudadas Cáceres apresentou os maiores valores de intensidade máxima de precipitação pluvial, com exceção para as durações de 20 e $720 \mathrm{~min}$, nas quais os maiores valores de precipitação ocorreram em Cuiabá e Rondonópolis, respectivamente.

Fazendo-se uma comparação entre Cuiabá e Rondonópolis, pode-se verificar que Cuiabá possui maiores valores de intensidade de precipitação para chuvas de duração curta (10, 20 e $30 \mathrm{~min}$ ), enquanto para durações maiores (50, 60, 120, 180, 240, 360, 720 e $1440 \mathrm{~min}$ ) os maiores valores de intensidade de precipitação ocorrem em Rondonópolis.

Os valores de intensidade máxima média obtidos para os três municípios estudados foram, em geral, inferiores às médias obtidas nos Estado de Santa Catarina, Tocantins e Rio Grande do Sul nos trabalhos desenvolvidos por Cardoso et al. (1997), Silva et al. (2003) e Damé et al. (2006), respectivamente.

A análise das relações obtidas entre as três estações para os valores extremos de intensidade máxima média de precipitação pluvial, para cada duração, revela tendência de valores crescentes com o aumento na duração de precipitação pluvial, sendo a menor relação para as durações de 30 e 40 min e a maior para a duração de $720 \mathrm{~min}$.

Esta tendência indica que os erros advindos da estimativa da intensidade máxima média para determinada localidade considerando os dados de outra localidade, tendem a crescer com o aumento na duração, fato este evidenciado também a partir da análise dos coeficientes de variação $(\mathrm{CV})$ obtidos para as diversas durações, na qual se verificam maiores valores de CV com o aumento da duração da precipitação pluvial (Tabela 2).

Face às grandes diferenças observadas entre as intensidades máximas médias de precipitação pluvial obtidas nas três estações para cada duração, sobretudo as maiores constata-se a necessidade de obtenção de equações que representem as condições das chuvas intensas para a localidade de interesse.

De posse dos dados de precipitação e intensidade de precipitação, a função de Gumbel (modelo teórico de probabilidade) foi ajustada aos dados de cada estação e se mostrou adequada na estimativa da precipitação máxima avaliada pelo teste de Kolmogorov-Smirnov para um nível de significância de 5\% de probabilidade.

A Tabela 3 apresenta as equações de chuvas intensas ajustadas pelos métodos de análise de pluviogramas, desagregação de chuvas de 24 h e Bell para os municípios de Cáceres, Cuiabá e Rondonópolis. 
Tabela 2. Intensidades máximas de precipitação $\left(\mathrm{mm} \mathrm{h}^{-1}\right)$ para as durações selecionadas para três municípios do Estado $M$ ato $\mathrm{G}$ rosso, pelo método de análise de pluviogramas

\begin{tabular}{|c|c|c|c|c|c|c|c|c|c|c|c|c|}
\hline \multirow{2}{*}{ Ano } & \multicolumn{12}{|c|}{ Duração (min) } \\
\hline & 10 & 20 & 30 & 40 & 50 & 60 & 120 & 180 & 240 & 360 & 720 & 1440 \\
\hline \multicolumn{13}{|c|}{ Cáceres } \\
\hline 1990 & 80,88 & 70,41 & 57,46 & 44,34 & 36,47 & 30,45 & 15,38 & 10,47 & 8,05 & 1,75 & 0,00 & 0,00 \\
\hline 1991 & 94,50 & 63,75 & 47,30 & 38,70 & 41,42 & 46,72 & 36,01 & 24,63 & 18,91 & 12,74 & 0,00 & 0,00 \\
\hline 1992 & 60,24 & 51,15 & 40,42 & 34,38 & 34,63 & 28,86 & 19,25 & 15,24 & 12,22 & 8,68 & 3,25 & 0,00 \\
\hline 1993 & 71,40 & 55,32 & 45,00 & 52,31 & 45,12 & 39,00 & 21,63 & 15,42 & 12,02 & 8,20 & 1,93 & 0,00 \\
\hline 1994 & 49,62 & 32,91 & 40,92 & 64,28 & 71,28 & 67,94 & 17,93 & 12,82 & 10,36 & 7,08 & 4,27 & 0,00 \\
\hline 1995 & 45,90 & 43,11 & 58,44 & 58,13 & 51,38 & 47,26 & 29,36 & 21,47 & 16,17 & 10,81 & 3,07 & 0,00 \\
\hline 1997 & 36,06 & 38,22 & 41,36 & 43,14 & 40,96 & 36,51 & 21,18 & 19,02 & 15,53 & 7,14 & 3,27 & 0,00 \\
\hline 1998 & 48,12 & 56,67 & 49,16 & 39,89 & 42,50 & 41,46 & 43,66 & 30,67 & 23,69 & 8,98 & 4,60 & 0,00 \\
\hline 1999 & 31,32 & 30,42 & 22,32 & 22,98 & 33,60 & 36,64 & 31,58 & 22,22 & 17,38 & 11,92 & 1,60 & 0,00 \\
\hline 2001 & 31,32 & 30,15 & 45,88 & 43,35 & 40,46 & 35,69 & 29,65 & 23,55 & 18,68 & 12,82 & 2,52 & 0,00 \\
\hline 2003 & 30,30 & 30,09 & 22,00 & 17,87 & 16,39 & 14,65 & 11,21 & 13,63 & 20,38 & 17,01 & 9,32 & 0,00 \\
\hline 2005 & 78,00 & 63,42 & 45,20 & 39,11 & 43,84 & 47,91 & 30,35 & 26,26 & 22,79 & 20,35 & 1,16 & 0,00 \\
\hline Média & 54,81 & 47,14 & 42,96 & 41,54 & 41,51 & 39,42 & 25,60 & 19,62 & 16,35 & 10,62 & 2,92 & 0,00 \\
\hline $\mathrm{DP}$ & 21,96 & 14,81 & 11,30 & 13,12 & 12,69 & 12,93 & 9,39 & 6,18 & 4,92 & 4,88 & 2,50 & 0,00 \\
\hline $\mathrm{CV}$ & 40,06 & 31,42 & 26,29 & 31,59 & 30,57 & 32,81 & 36,67 & 31,49 & 30,07 & 45,97 & 85,70 & \\
\hline Relação mx/mn & 3,12 & 2,34 & 2,66 & 3,58 & 4,35 & 4,64 & 3,90 & 2,93 & 2,94 & 11,63 & & \\
\hline \multicolumn{13}{|c|}{ Cuiabá } \\
\hline 1972 & 110,94 & 67,41 & 46,44 & 44,97 & 41,51 & 36,32 & 17,62 & 13,75 & 10,60 & 8,28 & 3,91 & 0,00 \\
\hline 1973 & 54,00 & 47,19 & 40,78 & 33,80 & 34,70 & 34,29 & 24,28 & 17,25 & 14,45 & 8,87 & 1,48 & 0,00 \\
\hline 1974 & 59,88 & 52,26 & 53,02 & 51,27 & 43,58 & 43,89 & 34,68 & 23,33 & 19,82 & 5,82 & 1,85 & 0,00 \\
\hline 1975 & 85,02 & 70,68 & 58,16 & 52,26 & 48,78 & 42,95 & 22,47 & 15,69 & 13,04 & 8,98 & 3,75 & 0,00 \\
\hline 1976 & 56,58 & 38,43 & 44,98 & 42,44 & 35,20 & 29,69 & 38,69 & 19,80 & 16,72 & 12,17 & 4,96 & 0,00 \\
\hline 1977 & 123,72 & 71,37 & 58,54 & 58,89 & 53,10 & 44,65 & 17,49 & 12,04 & 10,31 & 6,57 & 3,43 & 0,00 \\
\hline 1985 & 24,00 & 33,51 & 26,80 & 30,03 & 29,72 & 28,21 & 16,13 & 10,84 & 8,14 & 5,53 & 3,75 & 0,00 \\
\hline 1990 & 16,38 & 9,57 & 6,38 & 4,79 & 3,83 & 3,19 & 0,00 & 0,00 & 0,00 & 0,00 & 0,00 & 0,00 \\
\hline 1991 & 41,64 & 48,90 & 52,42 & 54,42 & 59,15 & 49,83 & 26,47 & 18,35 & 14,12 & 9,48 & 0,33 & 0,00 \\
\hline 1992 & 147,00 & 102,99 & 77,78 & 58,46 & 46,76 & 38,98 & 19,51 & 17,46 & 14,50 & 10,17 & 2,90 & 0,00 \\
\hline 1993 & 54,72 & 48,60 & 40,04 & 31,50 & 25,98 & 21,92 & 14,60 & 12,93 & 10,29 & 2,81 & 0,33 & 0,00 \\
\hline 1994 & 59,88 & 9,39 & 8,62 & 8,33 & 7,69 & 6,69 & 3,94 & 2,65 & 2,00 & 1,34 & 0,00 & 0,00 \\
\hline 1995 & 12,96 & 32,97 & 40,30 & 40,05 & 38,02 & 36,01 & 19,38 & 13,63 & 0,32 & 0,00 & 0,00 & 0,00 \\
\hline 1996 & 36,42 & 40,71 & 39,92 & 30,59 & 26,98 & 23,54 & 11,24 & 7,55 & 5,77 & 3,89 & 0,00 & 0,00 \\
\hline 1997 & 23,22 & 34,71 & 28,36 & 29,78 & 29,63 & 25,28 & 15,45 & 12,06 & 8,04 & 5,49 & 0,00 & 0,00 \\
\hline 1998 & 20,10 & 20,07 & 20,56 & 24,45 & 16,14 & 14,48 & 10,24 & 7,47 & 5,94 & 4,10 & 0,00 & 0,00 \\
\hline 1999 & 115,26 & 62,04 & 68,38 & 64,49 & 52,38 & 43,66 & 22,19 & 15,31 & 11,50 & 7,69 & 0,30 & 0,00 \\
\hline 2003 & 60,00 & 60,54 & 62,22 & 64,83 & 57,18 & 48,34 & 26,01 & 18,15 & 14,03 & 0,00 & 0,00 & 0,00 \\
\hline Média & 61,21 & 47,30 & 42,98 & 40,30 & 36,13 & 31,77 & 18,91 & 13,24 & 9,98 & 5,62 & 1,50 & \\
\hline $\mathrm{DP}$ & 40,00 & 23,43 & 19,45 & 17,68 & 16,11 & 13,82 & 9,57 & 5,95 & 5,56 & 3,75 & 1,78 & \\
\hline CV & 65,35 & 49,53 & 45,26 & 43,88 & 44,58 & 43,49 & 50,63 & 44,93 & 55,75 & 66,71 & 118,42 & \\
\hline Relação mx/mn & 11,34 & 10,97 & 12,19 & 13,53 & 7,69 & 15,62 & & & & & & \\
\hline \multicolumn{13}{|c|}{ Rondonópolis } \\
\hline 1992 & 57,36 & 42,78 & 43,38 & 43,82 & 40,64 & 34,94 & 18,90 & 17,47 & 14,25 & 13,20 & 2,90 & 0,00 \\
\hline 1993 & 28,20 & 39,93 & 43,02 & 38,87 & 34,79 & 30,10 & 15,73 & 17,51 & 13,38 & 8,93 & 2,61 & 0,00 \\
\hline 1995 & 41,70 & 36,57 & 33,10 & 39,32 & 39,72 & 41,15 & 34,23 & 23,67 & 8,31 & 9,26 & 7,47 & 0,00 \\
\hline 1997 & 34,32 & 35,70 & 34,52 & 26,25 & 23,50 & 20,91 & 14,85 & 10,16 & 7,66 & 5,11 & 3,99 & 0,00 \\
\hline 1998 & 58,26 & 28,77 & 38,88 & 46,52 & 43,40 & 37,57 & 21,31 & 15,82 & 14,26 & 10,28 & 3,64 & 0,00 \\
\hline 1999 & 35,94 & 47,94 & 44,30 & 40,29 & 51,82 & 44,66 & 16,24 & 20,34 & 17,61 & 12,82 & 4,08 & 0,00 \\
\hline 2005 & 59,58 & 54,33 & 54,90 & 47,24 & 40,22 & 34,57 & 17,81 & 12,14 & 8,42 & 5,62 & 2,64 & 0,00 \\
\hline Média & 45,05 & 40,86 & 41,73 & 40,33 & 39,16 & 34,84 & 19,86 & 16,73 & 11,98 & 9,32 & 3,90 & 0,00 \\
\hline $\mathrm{DP}$ & 13,10 & 8,44 & 7,29 & 7,07 & 8,62 & 7,75 & 6,70 & 4,61 & 3,85 & 3,16 & 1,69 & 0,00 \\
\hline $\mathrm{CV}$ & 29,09 & 20,65 & 17,47 & 17,52 & 22,02 & 22,24 & 33,71 & 27,54 & 32,11 & 33,87 & 43,29 & \\
\hline CV médio & 40,06 & 31,42 & 26,29 & 31,59 & 30,57 & 32,81 & 36,67 & 31,49 & 30,07 & 45,97 & 85,70 & \\
\hline Relação mx/mn & 2,11 & 1,89 & 1,66 & 1,80 & 2,21 & 2,14 & 2,31 & 2,33 & 2,30 & 2,58 & 2,86 & \\
\hline
\end{tabular}

DP - Desvio Padrão; CV - Coeficiente de Variação; Relação mx/mn - Relação entre valor máximo e mínimo de intensidade de precipitação; CV médio - Coeficiente de Variação médio

As equações geradas com os dados obtidos pelos métodos da análise de pluviogramas, desagregação de chuvas de $24 \mathrm{~h}$ e Bell, apresentaram bom ajuste, com valores de $\mathrm{r}^{2}$ acima de $94 \%$ e todos os parâmetros significativos a nível de $1 \%$ de probabilidade nos três municípios.
Pela análise de pluviogramas verifica-se que o valor do coeficiente $\mathrm{K}$ variou de 1382.43 a 7589.68 , relativos às estações de Cuiabá e Rondonópolis, respectivamente. O coeficiente a variou de 0.15 a 0.30 , relativos às estações de Rondonópolis e Cuiabá, respectivamente; o coeficiente b variou de 27.71 a 108.04, relativos às estações de Cuiabá e Rondonópolis, 
Tabela 3. Equações de chuvas intensas com os respectivos coeficientes estatísticos ajustados para os municípios Cáceres, Cuiabá e Rondonópolis, obtidos pelo método de Análise de Pluviogramas, D esagregação de Chuvas de 24 h e Bell

\begin{tabular}{|c|c|c|c|c|c|c|}
\hline \multirow{3}{*}{ Municípios } & \multicolumn{6}{|c|}{ Métodos } \\
\hline & \multicolumn{2}{|l|}{ Análise de pluviogramas } & \multicolumn{2}{|c|}{ Desagregação de chuvas de $24 \mathrm{~h}$} & \multicolumn{2}{|l|}{ Bell } \\
\hline & Equação & $\mathbf{R}^{2}$ & Equação & $\mathbf{R}^{2}$ & Equação & $\mathbf{R}^{2}$ \\
\hline Cáceres & $\mathrm{i}_{\mathrm{m}}=\frac{5378,867 \times \mathrm{TR}^{0,215101}}{(\mathrm{t}+93,24403)^{1,036769}}$ & 95,9 & $\mathrm{i}_{\mathrm{m}}=\frac{2031,858 \times \mathrm{TR}^{0,224841}}{(\mathrm{t}+13,23087)^{0,958269}}$ & 97,9 & $\mathrm{i}_{\mathrm{m}}=\frac{531,5929 \times \mathrm{TR}^{0,228262}}{(\mathrm{t}+4,184484)^{0,670812}}$ & 99,1 \\
\hline Cuiabá & $\mathrm{i}_{\mathrm{m}}=\frac{1382,435 \times \mathrm{TR}^{0,300247}}{(\mathrm{t}+27,71088)^{0,931493}}$ & 94,0 & $\mathrm{i}_{\mathrm{m}}=\frac{288,468 \times \mathrm{TR}^{0,387949}}{(\mathrm{t}-1,58582)^{0,755563}}$ & 94,0 & $\mathrm{i}_{\mathrm{m}}=\frac{416,7657 \times \mathrm{TR}^{0,232267}}{(\mathrm{t}+4,184481)^{0,670812}}$ & 99,1 \\
\hline Rondonópolis & $\mathrm{i}_{\mathrm{m}}=\frac{7589,682 \times \mathrm{TR}^{0,151341}}{(\mathrm{t}+108,0431)^{1,09192}}$ & 96,8 & $\mathrm{i}_{\mathrm{m}}=\frac{488,2158 \times \mathrm{TR}^{0,22236}}{(\mathrm{t}+4,184481)^{0,670812}}$ & 98,4 & $\mathrm{i}_{\mathrm{m}}=\frac{1798,665 \times \mathrm{TR}^{0,197914}}{(\mathrm{t}+13,2309)^{0,958269}}$ & 99,2 \\
\hline
\end{tabular}

respectivamente, e o coeficiente c variou de 0.93 a 1.09 , para as estações de Cuiabá e Rondonópolis, respectivamente.

Para as três estações estudadas os menores valores do coeficiente $\mathrm{b}$ estão relacionados aos maiores valores do coeficiente K. É importante ressaltar que outras combinações de coeficientes podem ser obtidas para a relação entre intensidade, duração e frequência, sem causar perda significativa na precisão dos resultados, em que um exemplo é o estudo realizado por Silveira (2006), no qual foram obtidos valores diferentes dos coeficientes para a localidade de Cuiabá utilizando-se a metodologia de Análise de Pluviogramas.

Observa-se, ainda, que os valores dos parâmetros $(\mathrm{K}, \mathrm{a}, \mathrm{b}$, c) de ajuste das equações variaram bastante entre as estações evidenciando a necessidade da determinação dessas equações para cada localidade e, consequentemente, obtendo maior na segurança de dimensionamento e menor custos das obras hidráulicas, principalmente, hidroagrícolas.

A Tabela 4 apresenta o erro relativo médio (ERM) das equações de chuvas intensas obtidas pelos métodos alternativos para os municípios estudados. Fazendo um estudo comparativo dos métodos de Desagregação de Chuvas de 24 h e de Bell em relação ao método de Análise de Pluviogramas, tem-se que o método da Desagragação apresentou melhor desempenho do que de Bell para as estações Cáceres e Cuiabá, porém na estação Rondonópolis, o método de Bell apresentou melhor desempenho que o método de Desagregação.

Tabela 4. Erro médio relativo, local e média, produzidos pelos métodos de Bell e D esagregação de Chuvas de $24 \mathrm{~h}$ em relação ao método padrão (Análise de Pluviogramas)

\begin{tabular}{lrrrrr}
\hline \multirow{2}{*}{ Localidade } & \multicolumn{3}{c}{ Erro medio relativo (\%) } \\
\cline { 2 - 3 } & \multicolumn{3}{c}{ Bell } & & \multicolumn{2}{c}{ Desagregaçáo } \\
\cline { 2 - 2 } & Local & Médio & & Local & Médio \\
Cáceres & 22,79 & & 15,01 & \\
Cuiabá & 10,93 & 16,85 & & 4,90 & 14,69 \\
Rondonópolis & 16,82 & & 33,95 & \\
\hline
\end{tabular}

Entende-se, portanto, que não houve um método que se tenha sobressaído, no presente estudo. Esta inversão de desempenho dos métodos na estação Rondonópolis, pode estar associada a uma série de dados menos extensa, o que desfavoreceu o método de Desagregação de chuvas de 24 h, indicando que este método parece ser mais sensível ao tamanho da série, comparativamente com o método de Bell.

\section{CONCLusÕES}

1. Para as três localidades estudadas a distribuição de Gumbel mostrou-se adequada na estimativa dos valores de intensidade máxima média “ $\mathrm{i}_{\mathrm{m}}$ ” a nível de significância de $5 \%$, pelo teste de Kolmogorov-Smirnov.

2. Os parâmetros das equações intensidade-duraçãofrequência, apresentaram alta variabilidade independente do método de obtenção dos dados de intensidade de precipitação.

3. Dentre as metodologias alternativas na determinação das precipitações máximas para diferentes durações e período de retorno, o método da Desagregação apresentou melhor desempenho global em comparação com o método de Bell.

4. O método de desagregação mostrou-se mais sensível ao tamanho da série comparativamente com o método de Bell, e desta forma, o seu desempenho na estimativa da intensidade máxima de precipitação foi pior do que o método de Bell para o município de Rondonópolis (município com menor série de dados - 7 anos).

\section{LITERATURA CITADA}

Back. A. J. Relationships between heavy precipitations of different durations in the municipality of Urussanga, SC. Revista Brasileira de Engenharia Agrícola e Ambiental, v.13, p.170-175, 2008.

Bertoni, J. C.; Tucci, E. M. Precipitação. In: Tucci, C. E. M. (Org.) Hidrologia. Porto Alegre: EDUSP, ABRH, 1993. Cap.4, p.177-231.

Cardoso, C. O.; Ullmann, M. N.; Bertol, I. Análise de chuvas intensas a partir da desagregação das chuvas diárias de Lages e de Campos Novos (SC). Revista Brasileira de Ciência do Solo, v.22, p.131-140, 1988.

Cecílio, R. A.; Pruski, F. F. Interpolação dos parâmetros da equação de chuvas intensas com uso do inverso de potências da distância. Revista Brasileira de Engenharia Agrícola e Ambiental, v.7, p.501-504, 2003. 
DAEE-CETESB - Departamento de Águas e Energia Elétrica do Estado de São Paulo - Companhia de Tecnologia de Saneamento Ambiental. Drenagem urbana - Manual de projeto. 3.ed. São Paulo: CETESB, 1986. 464p.

Damé, R. C. F.; Pedrotti, C. B. M.; Cardoso, M. A. G.; Silveira, C. P.; Duarte, L. A.; Ávila, M. S. V.; Moreira, A. C. Comparação entre curvas intensidade-duração-frequência de ocorrência de precipitação obtidas a partir de dados pluviográficos com àquelas estimadas por técnicas de desagregação de chuva diária. Revista Brasileira de Agrociência, v.12, p.505$509,2006$.

Damé, R. de C. F.; Teixeira, C. F. A.; Terra, V. S. S. Comparação de diferentes metodologias para estimativa de curvas intensidade-duração-frequência para Pelotas - RS. Engenharia Agrícola, v.28, p.10-15, 2008.

Leopoldo, P. R.; Sansigolo, C. A.; Martins, D. Análise estatística das intensidades e precipitações máximas de Botucatu. Irrigação e Tecnologia Moderna, v.16, p.11-14, 1984.

Mello, C. R.; Ferreira, D. F.; Silva, A. M.; Lima, J. M. Análise de modelos matemáticos aplicados ao estudo de chuvas intensas. Revista Brasileira de Ciência do Solo, v.25, p.693698, 2001.

Mello, C. R. de; Silva, A. M.; Lima, J. M.; Ferreira, D. F.; Oliveira, M. S. Modelos matemáticos para predição da chuva de projeto para regiões do estado de Minas Gerais. Revista Brasileira de Engenharia Agrícola e Ambiental, v.7, p.121$128,2003$.
Oliveira, L. F. C. de; Antonini, J. C. dos A.; Fioreze, A. P.; Silva, M. A. S. da. Métodos de estimativa de precipitação máxima para o Estado de Goiás. Revista Brasileira de Engenharia Agrícola e Ambiental, v.12, p. 620-625, 2008a.

Oliveira, L. F. C. de; Antonini, J. C. dos A.; Griebeler, N. P. Estimativas de chuvas intensas para o Estado de Goiás. Engenharia Agrícola, v.28, p.22-33, 2008b.

Oliveira, L. F. C. de; Cortês, F. C.; Wehr, T. R.; Borges, L. B.; Sarmento, P. H. P.; Griebeler, N. P. Intensidade-duraçãofrequência de chuvas intensas para algumas localidades no Estado de Goiás e Distrito Federal. Pesquisa Agropecuária Tropical, v.35, p.13-18, 2005.

Oliveira, L. F. C. de; Pruski, F. F. Modelos para estimar as perdas de solo e água e transporte de solutos. 1.ed. Minas Gerais: Viçosa, 1996. 70p.

Silva, D. D.; Pereira, S. B.; Pruski, F. F.; Gomes Filho, R. R.; Lana, A. M. Q.; Baena, L. G. N. Equações de intensidadeduração-frequência da precipitação pluvial para o Estado de Tocantins. Engenharia na Agricultura, v.11, p. 1-8, 2003.

Silveira, A. Curva intensidade-duração-frequência das precipitações extremas para o município de Cuiabá. Cuiabá: Tecnosan Engenharia, 2006. 12p.

Svensson, C.; Clarke, R. T.; Jones, D. A. An experimental comparison of methods for estimating rainfall intensityduration-frequency relations from fragmentary records. Journal of Hydrology, v.341, p.79-89, 2007. 